du procureur général de la couronne près le ministère de la guerre ; reconnaissant que l'œuvre humanitaire à laquelle la Commission portugaise désire coopérer, vient en aide à l'idée de diminuer autant que possible les maux de la guerre, et s'accorde avec la Convention internationale de Genève, du 22 aoùt 1864.

Nous approuvons les Statuts qui seront promulgués avec le présent décret par le ministère secrétaire d'Etat pour les affaires de la guerre, et reconnaissons l'existence légale de la Commission, pour le but exclusif dont elle s'occupe en vertu desdits Statuts.

Le ministre secrétaire d'Etat pour les affaires de la guerre en sera informé et veillera à l'exécution du présent décret.

Au Palais, le 26 mai 1868. (Signé) Le Ror.

(Contre-signé) José Maria de Magalhaes.

\title{
STATUTS DE LA COMMISSION PORTUGAISE
}

Art. 1. Il est fondé à Lisbonne une Association, sous le nom de Commission portugaise de secours aux militaires blessés et malades en temps de guerre, pour concourir à l'œuvre humanitaire indiquée par ce titre, selon les vues de la Conférence européenne, réunie à Genève, en l'année 1863, lesquclles furent suivies de la Convention de Genève, du 22 aoùt 1864.

Art. 2. Cette Commission fonctionne sous les auspices du ministère dè la guerre et correspond avec le Comité international siégeant à Genève, mais sans être obligée par les délibérations de ce Comité.

Art. 3. La Commission forme le noyau et le centre des souscommissions qui pourront s'organiser dans le royaume de Portugal et ses dépendances, afin de développer son cuvre et d'atteindre le mieux possible le but qu'elle se propose.

Art. 4. Sont membres actifs de la Commission tous ses fondateurs, ainsi que les personnes qu'elle admet dans son sein; sont membres adhèrents ceux quĩ s'inscrivent comme souscripteurs pour une somme annuelle de 1200 réis au moins (fr. 6 environ).

Les dames peuvent faire partie de la Commission.

Art. 5. En temps ordinaire, la Commission recueille et capitalise des dons en argent, ou les emploie à l'achat d'objets utiles pour l'assistance qu'elle doit procurer; elle accepte et recueille les offres de toute nature et de toute espèce ayant la même destination; elle 


\section{5}

s'occupe de tous les perfectionnements à introduire dans la manière de porter secours; elle correspond pour cela, non-seulement avec le Comité interuational de Genève, mais avec ceux de tous les autres pays; elle poursuit en un mot de toutes manières le but de son institution.

Art. 6. La Commission peut demander au Ministère de la guerre de vouloir bien se charger de la garde et de la conservation du matériel sanitaire acquis par elle.

Art. 7. Pour exercer la direction et les fonctions qui lui sont attribuées, la Commission nomme un président, un vice-président, -un secrétaire général, deux vice-secrétaires et un trésorier. Tous ces emplois sont gratuits et ne dispensent pas de la contribution.

Art. 8. En outre la Commission peut avoir autant de présidents honoraires qu'il y aura de personnes jugées dignes de cette distinction, par le fait de services rendus à l'œuvre charitable dont elle s'occupe.

Art. 9. La Commission se réunit à des époques indéterminées pour la décision des affaires qui lui sont soumises; elle élit de nouveaux fonctionnaires lorsqu'un ou plusieurs emplois se trouvent vacants. De plus, il y a, au mois de janvier de chaque année, une assemblée régulière, dans laquelle on prend connaissance de tout ce qui est survenu d'important pendant l'année précédente,

Art. 10. La Commission publie tous les deux ans, ou plus souvent s'il y a lieu, un rapport sur ses travaux.

Art. 11. Si quelque éventualité déterminait la Commission à ne pas continuer ses travaux, tout ce qu'elle posséderait serait remis au Ministère de la guerre, pour être affecté au service sanitaire de l'armée.

Au Palais, le 26 mai 1868.

(Signé) José Maria de Magalhaes. 